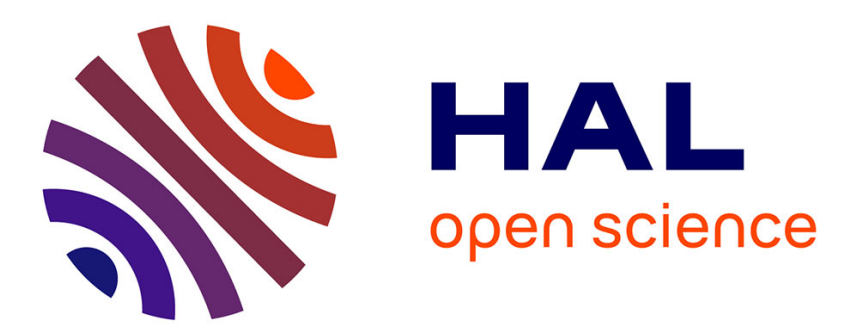

\title{
The Symmetric and Asymmetric Choquet integrals on finite spaces for decision making
}

Michel Grabisch, Christophe Labreuche

\section{To cite this version:}

Michel Grabisch, Christophe Labreuche. The Symmetric and Asymmetric Choquet integrals on finite spaces for decision making. Statistical Papers, 2002, 43 (1), pp.37-52. 10.1007/s00362-001-0085-4 . halshs-00273184

\section{HAL Id: halshs-00273184 \\ https://shs.hal.science/halshs-00273184}

Submitted on 14 Apr 2008

HAL is a multi-disciplinary open access archive for the deposit and dissemination of scientific research documents, whether they are published or not. The documents may come from teaching and research institutions in France or abroad, or from public or private research centers.
L'archive ouverte pluridisciplinaire HAL, est destinée au dépôt et à la diffusion de documents scientifiques de niveau recherche, publiés ou non, émanant des établissements d'enseignement et de recherche français ou étrangers, des laboratoires publics ou privés. 
Statistical Letters manuscript No.

(will be inserted by the editor)

\title{
The Symmetric and Asymmetric Choquet integrals on finite spaces for decision making ${ }^{\star}$
}

\author{
Michel GRABISCH ${ }^{\star \star 1}$, Christophe LABREUCHE ${ }^{2}$ \\ 1 LIP6, University of Paris VI, 4 Place Jussieu, 75252 Paris, France \\ email Michel.Grabisch@lip6.fr \\ 2 Thomson-CSF, Corporate Research Laboratory, Domaine de Corbeville, 91404 \\ Orsay Cedex, France \\ email labreuche@lcr.thomson-csf.com
}

Received: date / Revised version: date

\begin{abstract}
In this paper, we give a mathematical analysis of symmetric and asymmetric Choquet integrals in the view of decision making in a finite setting. These integrals present two ways of dealing with negative integrands. The analysis is done with the aid of the Möbius and interaction transforms, this last one having an interesting interpretation in multicriteria decision making (MCDM). The last part of the paper shows the application of these two integrals in MCDM.
\end{abstract}

\section{Introduction}

The usage of capacities and the Choquet integral [2] as a generalization of the notion of probability and expected value has recently widened, due to its connection with the field of imprecise probabilities [33], and to its contribution in decision making. In this last field, the pioneering work of Schmeidler $[24,25]$, followed by many others, has shown that the Choquet expected utility model was able to bring a more flexible tool to mimic human decision making.

Another important field of decision making is multicriteria decision making, which is connected to multiattribute utility theory [19], and also to multidimensional measurement theory [20]. Recently, the Choquet integral has been used in this field as a powerful way to aggregate criteria $[9,16$,

\footnotetext{
* This paper is based on a preliminary and short version published at the IPMU'2000 Conference [15].

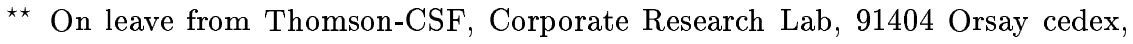
France
} 
17]. This common use of the Choquet integral in two different paradigms and indeed in two distinct scientific communities-, is not surprising if one considers the formal parallel between decision under uncertainty and multicriteria decision making, where criteria play the role of states of nature (see $[6])$.

The use of Choquet integral in multicriteria decision making (MCDM) has led to the notion of interaction transform (or representation) $[11,5]$ of a capacity (also called fuzzy measure [28], or non-additive measure [4]), a concept generalizing the Shapley value [27]. In a problem of decision making under multiple criteria, the importance of a criterion to make decision is a central concern, as well as the notion of dependent criteria. These notions have never been properly defined, till the pioneering works of Murofushi, who proposed to use the Shapley value as a mean to define the importance of a criterion [22], and the concept of interaction index for a pair of criteria [23], based on considerations borrowed from multiattribute utility theory. The interaction transform is a generalization of both the Shapley value and the interaction index of Murofushi and Soneda, which offers a very clear and intuitive way to understand the behaviour in decision induced by a given capacity.

Another important concept, which is a direct consequence of the introduction of the interaction transform, is the notion of $k$-additive measure (capacity) [11]. $k$-additive measures are simply capacities for which the interaction transform vanishes for subsets of more than $k$ elements. In other words, there is no interaction among criteria for coalitions of more than $k$ criteria. 1-additive measures are simply additive measures, i.e. probability measures.

In decision under risk or uncertainty, prospect theory [18] models decision behaviours when one distinguishes between positive values of utility, called "gains", and negative ones, called "losses", in accordance with observations done by psychologists. It has been generalized later under the name "cumulative prospect theory" (CPT) [30], so that its restriction to gains coincide with the Choquet expected utility model. In fact, the integral underlying the CPT model is called the symmetric integral by Denneberg [4], an integral introduced already by Šipoš [31] in 1979. It is different from the usual definition of the Choquet integral for real-valued functions, which is called "asymmetric integral" by Denneberg. These two names come from the characteristic property that for any capacity $v$ and any act $f$, $\mathcal{C}_{v}(-f)=-\mathcal{C}_{\bar{v}}(f)$, and $\check{\mathcal{C}}_{v}(-f)=-\check{\mathcal{C}}_{v}(f)$, where $\mathcal{\mathcal { C }}_{v}, \check{\mathcal{C}}_{v}$ denote respectively the Choquet (asymmetric) and Šipoš (symmetric) integrals with respect to capacity $v$, and $\bar{v}$ is the conjugate capacity. Recently, the authors have studied the use of symmetric integral in the field of MCDM $[15,16]$, and thanks to the interaction transform, they revealed the fundamental difference in behaviour with the asymmetric (usual) Choquet integral model.

The aim of this paper is to give a comparative analysis of both integrals, in the light of the interaction and Möbius transforms, keeping in mind their application in decision making, more particularly multicriteria 
decision making. Therefore, we will restrict to the finite case (finite number of states of nature and of criteria). The paper should offer to the reader both a comparative analysis of properties in a finite setting, and a clear understanding of the decision behaviour implied by these models. We begin by a introduction of necessary notions and notations.

In the sequel, $\wedge, \vee$ denote min and max respectively.

\section{Basic definitions and notations}

Let $N=\{1, \ldots, n\}$ be a finite set, representing the index set of states of nature or criteria, etc. A capacity or fuzzy measure, non-additive measure on $N$ is any set function $v: \mathcal{P}(N) \longrightarrow \mathbb{R}^{+}$such that $v(\emptyset)=0$ and $A \subset B \subset N$ implies $v(A) \leq v(B)$ (monotonicity). $v\left(A^{c}\right)$.

The conjugate capacity of $v$, denoted $\bar{v}$, is defined by $\bar{v}(A)=v(N)-$

Particular capacities of interest are the unanimity games $u_{A}$, with $A \subset$ $N$. They are defined as:

$$
u_{A}(B):= \begin{cases}1, & \text { if } B \supset A \\ 0, & \text { otherwise. }\end{cases}
$$

We introduce two useful linear transformations of capacities (see $[5,13]$ for a full presentation of this topic).

Definition 1 Let $v$ be a capacity. For all $A \subset N$ :

- the Möbius transform of $v$ is given by:

$$
m^{v}(A):=\sum_{B \subset A}(-1)^{|A|-|B|} v(B) .
$$

- the interaction transform is given by:

$$
I^{v}(A):=\sum_{B \subset N \backslash A} \xi_{n}(|B|,|A|) \sum_{C \subset A}(-1)^{|A|-|C|} v(C \cup B),
$$

with $\xi_{n}(k, l)=\frac{(n-k-l) ! k !}{(n-l+1) !}$.

When possible, the superscripts $v$ will be omitted.

A third linear transform is the co-Möbius transform [13]. Since its interest is more technical, but very useful in the proofs, we put its definition and related properties in the appendix.

It is known that any capacity can be written as a linear combination of unanimity games thanks to the Möbius transform. Specifically:

$$
v(A)=\sum_{B \subset N} m(B) u_{B}(A)
$$


In other words, the unanimity games form a basis for the capacities, and the Möbius transform is the coordinate vector of the capacity.

Concerning the interaction transform, its meaning in decision making will become clear in Section 4. However, on a formal point of view, it is a generalization of the Shapley value [27], and of the interaction index $I_{i j}$ defined for a pair of criteria $i, j$ by Murofushi and Soneda [23]. The Shapley value of $v$ is the vector $\phi^{v}:=\left[\phi_{1}^{v} \cdots \phi_{n}^{v}\right]$ defined $\forall i \in N$ by:

$$
\phi_{i}^{v}:=\sum_{K \subset N \backslash i} \xi_{n}(|K|, 1)[v(K \cup\{i\})-v(K)] .
$$

The interaction index $I_{i j}$ is defined by:

$$
\begin{aligned}
I_{i j}^{v}:= & \sum_{K \subset N \backslash\{i, j\}} \xi_{n}(|K|, 2)[v(K \cup\{i, j\})-v(K \cup\{i\})- \\
& v(K \cup\{j\})+v(K)], \quad \forall i, j \in N .
\end{aligned}
$$

Clearly, $\phi_{i}^{v}=I^{v}(\{i\})$, and $I_{i j}^{v}=I^{v}(\{i, j\})$.

These transformations are invertible and can be composed. In the sequel, we will need the following formula:

$$
m(A)=\sum_{B \subset N \backslash A} B_{|B|} I(A \cup B)
$$

with $B_{k}$ the Bernoulli numbers, defined by the recurrence relation

$$
B_{k}:=-\sum_{l=0}^{k-1} \frac{B_{l}}{k-l+1}\left(\begin{array}{l}
k \\
l
\end{array}\right), k>0,
$$

and $B_{0}=1$. We will need also the coefficients $\beta_{k}^{l}$ defined by

$$
\beta_{k}^{l}:=\sum_{j=0}^{k}\left(\begin{array}{l}
k \\
j
\end{array}\right) B_{l-j}, \quad k, l=0,1,2, \ldots .
$$

We introduce now integrals with respect to capacities. As we are in the finite case, we assimilate integrand functions (resp. positive integrand functions) with vectors in $\mathbb{R}^{n}$ (resp. $\mathbb{R}_{+}^{n}$ ).

Definition 2 Let $v$ be a capacity, and $a=\left(a_{1}, \ldots, a_{n}\right) \in \mathbb{R}_{+}^{n}$. The Choquet integral of a with respect to $v$ is defined by:

$$
\mathcal{C}_{v}\left(a_{1}, \ldots, a_{n}\right):=\sum_{i=1}^{n}\left[a_{(i)}-a_{(i-1)}\right] v\left(A_{(i)}\right),
$$

where $\cdot_{(i)}$ indicates a permutation on $N$ so that $a_{(1)} \leq a_{(2)} \leq \cdots \leq a_{(n)}$, and $A_{(i)}:=\{(i), \ldots,(n)\}$. Also $a_{(0)}:=0$. 
We extend the definition to real integrand functions. For any $a=\left(a_{1}, \ldots, a_{n}\right) \in \mathbb{R}^{n}$, let us introduce $a^{+}, a^{-}$, the positive and the negative parts of $a$, that is $a^{+}=\left(a_{1}^{+}, \ldots, a_{n}^{+}\right), a^{-}=\left(a_{1}^{-}, \ldots, a_{n}^{-}\right), a_{i}^{+}=a_{i} \vee 0$, $a_{i}^{-}=-a_{i} \vee 0$. According to Denneberg [4], two integrals can be defined.

- the symmetric integral, denoted $\check{\mathcal{C}}_{v}$, is defined by:

$$
\check{\mathcal{C}}_{v}(a)=\mathcal{C}_{v}\left(a^{+}\right)-\mathcal{C}_{v}\left(a^{-}\right) .
$$

It was originally introduced by Šipoš [31].

- the asymmetric integral, denoted $\mathcal{C}_{v}$, is defined by:

$$
\check{\mathcal{C}}_{v}(a)=\mathcal{C}_{v}\left(a^{+}\right)-\mathcal{C}_{\bar{v}}\left(a^{-}\right) .
$$

It is the usual definition of the Choquet integral for real integrands, hence the notation.

These two integrals take their name from the following property. For any $a \in \mathbb{R}^{n}$,

$$
\begin{aligned}
\mathcal{C}_{v}(-a) & =-\mathcal{C}_{\bar{v}}(a) \\
\check{\mathcal{C}}_{v}(-a) & =-\check{\mathcal{C}}_{v}(a) .
\end{aligned}
$$

\section{Comparison between the symmetric and asymmetric integrals}

Our aim in this section is to give a clear understanding of the differences and similarities between the two integrals. We begin by rewriting (8) for the two integrals. We easily get, for any $a \in \mathbb{R}^{n}$ :

$$
\begin{aligned}
\mathcal{C}_{v}(a) & =a_{(1)}+\sum_{i=2}^{n}\left[a_{(i)}-a_{(i-1)}\right] v(\{(i), \ldots,(n)\}) \\
\check{\mathcal{C}}_{v}(a)= & \sum_{i=1}^{p-1}\left[a_{(i)}-a_{(i+1)}\right] v(\{(1), \ldots,(i)\})+a_{(p)} v(\{(1), \ldots,(p)\}) \\
& +a_{(p+1)} v(\{(p+1), \ldots,(n)\})+\sum_{i=p+2}^{n}\left[a_{(i)}-a_{(i-1)}\right] v(\{(i), \ldots,(n)\})
\end{aligned}
$$

where ${ }_{(i)}$ indicates a permutation on $N$ so that $a_{(1)} \leq a_{(2)} \leq \cdots \leq a_{(p)}<$ $0 \leq a_{(p+1)} \leq \cdots \leq a_{(n)}$.

Next, we rewrite the above expression using the Möbius transform. We get (see [16] for a proof):

$$
\begin{aligned}
\mathcal{C}_{v}(a) & =\sum_{A \subset N} m(A) \bigwedge_{i \in A} a_{i}, \\
\check{\mathcal{C}}_{v}(a) & =\sum_{A \subset N} m(A)\left[\bigwedge_{i \in A} a_{i}^{+}-\bigwedge_{i \in A} a_{i}^{-}\right] \\
& =\sum_{A \subset N^{+}} m(A) \bigwedge_{i \in A} a_{i}+\sum_{A \subset N^{-}} m(A) \bigvee_{i \in A} a_{i},
\end{aligned}
$$


where $N^{+}:=\left\{i \in N \mid a_{i} \geq 0\right\}$ and $N^{-}=N \backslash N^{+}$.

We turn now to the expression in terms of interactions indices.

Proposition 1 Let $a \in \mathbb{R}^{n}$.

$$
\begin{aligned}
\mathcal{C}_{v}(a)= & \sum_{A \subset N}\left(\sum_{B \subset N \backslash A} B_{|B|} I^{+}(A \cup B)\right) \bigwedge_{i \in A} a_{i} \\
& +\sum_{A \subset N, A \neq \emptyset}(-1)^{|A|+1}\left(\sum_{B \subset N \backslash A} \beta_{|B|}^{|B|} I^{-}(A \cup B)\right) \bigvee_{i \in A} a_{i} \\
\check{\mathcal{C}}_{v}(a)= & \sum_{A \subset N^{+}}\left(\sum_{B \subset N \backslash A} B_{|B|} I^{+}(A \cup B)\right) \bigwedge_{i \in A} a_{i} \\
& +\sum_{A \subset N^{-}}\left(\sum_{B \subset N \backslash A} B B_{|B|} I^{+}(A \cup B)\right) \bigvee_{i \in A} a_{i} \\
& +\sum_{A \cap N^{+} \neq \emptyset}(-1)^{|A|+1}\left(\sum_{B \subset N \backslash A} \beta_{|B|}^{|B|} I^{-}(A \cup B)\right) \bigvee_{i \in A} a_{i} \\
& +\sum_{A \cap N^{-} \neq \emptyset}(-1)^{|A|+1}\left(\sum_{B \subset N \backslash A} \beta_{|B|}^{|B|} I^{-}(A \cup B)\right) \bigwedge_{i \in A} a_{i},
\end{aligned}
$$

where $I^{+}(A)=I(A)$ if $I(A)>0$ and 0 otherwise, and $I^{-}(A)=I(A)$ if $I(A)<0$ and 0 otherwise.

Proof: let $a \in \mathbb{R}^{n}$, and let $v$ fixed, with interaction index $I(A)$. We define the set functions $v^{+}, v^{-}$by their interaction indices:

$$
I^{v^{+}}(A)=\left\{\begin{array}{ll}
I(A), & \text { if } I(A)>0 \\
0, & \text { otherwise }
\end{array}, \quad I^{v^{-}}(A)= \begin{cases}I(A), & \text { if } I(A)<0 \\
0, & \text { otherwise }\end{cases}\right.
$$

so that, by linearity of $I$ w.r.t $v$, we have $v=v^{+}+v^{-}$. We start with the asymmetric Choquet integral. By linearity of the integral w.r.t the measure, $\mathcal{C}_{v}(a)=\mathcal{C}_{v^{+}}(a)+\mathcal{C}_{v^{-}}(a)$. Using (13) and (23), we have for all $a \in \mathbb{R}^{n}$ :

$$
\begin{aligned}
& \mathcal{C}_{v^{+}}(a)=\sum_{A \subset N} m^{v^{+}}(A) \bigwedge_{i \in A} a_{i} \\
& \mathcal{C}_{v^{-}}(a)=\sum_{A \subset N, A \neq \emptyset}(-1)^{|A|+1} \check{m}^{v^{-}}(A) \bigvee_{i \in A} a_{i}
\end{aligned}
$$

Using (5) and (22) leads directly to the desired result.

The case of the symmetric integral proceeds similarly: it suffices to write $\check{\mathcal{C}}_{v}(a)=\check{\mathcal{C}}_{v^{+}}(a)+\check{\mathcal{C}}_{v^{-}}(a)$, to express $\check{\mathcal{C}}_{v^{+}}$and $\check{\mathcal{C}}_{v^{-}}$by $(14)$ and $(24)$, and then to use (5) and (22) again.

We give now characterizations of the asymmetric and symmetric integrals. 
Proposition 2 [21, Theorem 6.1.1] Let us consider a functional $M_{v}: \mathbb{R}^{n} \longrightarrow$ $\mathbb{R}$ defined with respect to a capacity $v$. Then $M_{v}$ is the asymmetric Choquet integral $\mathcal{C}_{v}$ for all capacities $v$ on $N$, if and only if $M_{v}$ satisfies the following properties:

(i) $M_{v}$ is a linear function of $v$, i.e. $M_{v}=\sum_{A \subset N} f_{A} v(A)$, where the $f_{A}$ 's are $2^{n}$ functions from $\mathbb{R}^{n}$ to $\mathbb{R}$.

(ii) $M_{v}$ is non decreasing in each place.

(iii) $M_{v}$ is invariant to positive affine transformations:

$$
M_{v}\left(\alpha a_{1}+\beta, \ldots, \alpha a_{n}+\beta\right)=\alpha M_{v}\left(a_{1}, \ldots, a_{n}\right)+\beta, \forall \alpha>0, \forall \beta \in \mathbb{R}
$$

(iv) $M_{v}$ is an extension of $v: M_{v}\left(1_{A}, 0_{A^{c}}\right)=v(A), \forall A \subset N$.

Proposition 3 Let us consider a functional $M_{v}: \mathbb{R}^{n} \longrightarrow \mathbb{R}$ defined with respect to a capacity $v$. Then $M_{v}$ is the symmetric Choquet integral $\check{\mathcal{C}}_{v}$ for all capacities $v$ on $N$, if and only if $M_{v}$ satisfies the following properties:

(L) $M_{v}$ is a linear function of $v$, i.e. $M_{v}=\sum_{A \subset N} f_{A} v(A)$, where the $f_{A}$ 's are $2^{n}$ functions from $\mathbb{R}^{n}$ to $\mathbb{R}$.

(In) $M_{v}$ is non decreasing in each place.

(H) $M_{v}$ is homogeneous:

$$
\check{\mathcal{C}}_{v}\left(\alpha a_{1}, \ldots, \alpha a_{n}\right)=\alpha \check{\mathcal{C}}_{v}\left(a_{1}, \ldots, a_{n}\right), \forall \alpha \in \mathbb{R} .
$$

(Sh) $M_{v}$ is invariant to positive shifts, i.e. $\forall a \in\left(\mathbb{R}^{+}\right)^{n}, \forall \alpha \geq 0, M_{v}(a+\alpha)=$ $M_{v}(a)+\alpha$.

(Ind) $M_{v}$ satisfies independence between positive and negative parts, i.e. for any $a, b, c, d \in \mathbb{R}^{n}, a \geq 0, b \geq 0, c \leq 0, d \leq 0, \forall A \subset N$, we have

$$
M_{v}\left(a_{A}, c_{A^{c}}\right)-M_{v}\left(a_{A}, d_{A^{c}}\right)=M_{v}\left(b_{A}, c_{A^{c}}\right)-M_{v}\left(b_{A}, d_{A^{c}}\right)
$$

(Ext) $M_{v}$ is an extension of $v$.

Proof: the necessity part is obvious. Let us prove the sufficiency part. Let $a \in \mathbb{R}^{n}$, with $a^{+}, a^{-}$defined as before. We apply (Ind) to $a=a^{+}, b=0, c=$ $-a^{-}, d=0$, and $A=\left\{i \in N \mid a_{i} \geq 0\right\}$. Then

$$
\begin{gathered}
M_{v}\left(a_{A}^{+},-a_{A^{c}}^{-}\right)-M_{v}\left(a_{A}^{+},-0_{A^{c}}\right)= \\
M_{v}\left(0_{A},-a_{A^{c}}^{-}\right)-M_{v}\left(0_{A},-0_{A^{c}}\right)
\end{gathered}
$$

which leads to, using $(\mathrm{H})$,

$$
M_{v}(a)=M_{v}\left(a^{+}\right)-M_{v}\left(a^{-}\right) .
$$

Let us now restrict to $a \in \mathbb{R}_{+}^{n}$. Combining axioms (H) and (Sh) implies the invariance to positive affine transformations. This property combined with (L), (In) and (Ext) leads to the fact that $M_{v}$ restricted to $\mathbb{R}_{+}^{n}$ is a Choquet integral $\mathcal{C}_{v}$. Due to (19), we finally have for any $a \in \mathbb{R}^{n}$

$$
M_{v}(a)=\mathcal{C}_{v}\left(a^{+}\right)-\mathcal{C}_{v}\left(a^{-}\right)=\check{\mathcal{C}}_{v}(a) .
$$


The property (Ind) means that the difference $M_{v}\left(a_{A}, c_{A^{c}}\right)-M_{v}\left(a_{A}, d_{A^{c}}\right)$ does not depend on $a$ : positive scores and negative scores do not interact.

The above properties make clear the following. As remarked by Sugeno and Murofushi [29], due to properties (iii) and (H) of propositions 2 and 3 resp., the asymmetric integral maps an interval scale to an interval scale, i.e. a scale where only difference of numbers has a meaning. On the other hand, the symmetric integral maps a ratio scale (where also the ratio of numbers is meaningful) to a ratio scale. A ratio scale has a zero with a fixed position, while its position is arbitrary for a difference scale. Hence formulas (11) and (13) do not differentiate between negative and positive numbers, while the symmetric integral makes this distinction, as observed by psychologists. For a deeper study in the general framework of decision making, see [14].

\section{Application to decision under multiple criteria}

We devote the rest of the paper to the application of both integrals in decision making under multiple criteria. For a deeper view, see [16].

\subsection{Capacities in decision under multiple criteria}

We present in a simplified way the framework, avoiding intricacies. Let $N=\{1, \ldots, n\}$ be a set of criteria. Acts are denoted by points $x \in \mathbb{R}^{n}$, of which coordinates are the scores or utilities on each criterion for a given act (we suppose that utility scales have been built, and that commensurability issues have been solved, using suitable information from the decision maker). The goal is to build a (global) utility function $u: \mathbb{R}^{n} \longrightarrow \mathbb{R}$, which assigns to each act its evaluation.

Let us call any $A \subset N$ a coalition of criteria. We introduce the notion of importance of a coalition as follows.

Definition 3 Let $A \subset N$ be a coalition of criteria. The importance or power $\mu(A)$ of coalition $A$ for a given decision maker is defined as the global evaluation of the fictitious act $\left(1_{A}, 0_{A^{c}}\right)$, i.e. with $x_{i}=1$ if $i \in A$ and 0 otherwise.

$$
\mu(A):=u\left(1_{A}, 0_{A^{c}}\right), \quad \forall A \subset N,
$$

where $u$ denotes global utility.

Clearly, the importance of the empty coalition should be zero, and the importance of $N$ should be maximum. Also, it is natural to require that the importance should be non decreasing when adding a new criterion in a coalition. In other words, we see that $\mu$ can be taken as a capacity.

An important consequence of this definition is that the global utility function $u$ has to coincide with $\mu$ on all the vertices of $[0,1]^{n}$, and so is an 
extension of $\mu$ on the whole unit hypercube. From Propositions 2 and 3, we know that precisely the symmetric and asymmetric Choquet integrals possess this property of extension, so that they are suitable models for $u$. This kind of link between the capacity and the global utility function has been brought into light by Marichal [21], and is the fundamental axiom in his result of characterization of Choquet integral.

Also, the previous results (see especially Propositions 2 and 3) show clearly the difference between the asymmetric and symmetric integrals. As already mentionned, the main fact is that the asymmetric integral supposes an underlying interval scale (arbitrary zero), while the symmetric integral supposes a ratio scale with a fixed zero. In multicriteria decision making using bipolar scales (that is, with positive and negative numbers), the meaning of the zero is that of a neutral satisfaction: the act is neither good nor bad on this criterion. It is commonly admitted that human decision makers have a different behaviour when faced to acts which are good or bad on a given criterion, which advocates the presence of a ratio scale instead of an interval scale.

The following example illustrates this.

Example 1 We consider a scientifically oriented school, and three subjects Maths, Physics and Literature. Let us assume that the neutral value is 10, and suppose 2 students with the following marks:

\begin{tabular}{|l|l|l|l|}
\hline Student & Maths & Physics & Literature \\
\hline $\mathrm{A}$ & 16 & 12 & $* *$ \\
$\mathrm{~B}$ & 8 & 4 & $* *$ \\
\hline
\end{tabular}

The following behaviour may be observed: since there is some redundancy between Maths and Physics in the sense that they require more or less the same kind of ability, a medium score in one of the two can be compensated by a good score in the other subject. Thus, we can give 15 or 16 to $\mathrm{A}$ (provided the literature is not too bad). But if both are bad as for B, due to the importance of scientific subjects, one should not be tolerant with such a student, and something like 4 or 5 should be given as global evaluation.

This example shows that tolerance is observed when scores are good, and intolerance when scores are bad, exhibiting a symmetric behaviour. Of course, the converse of this example may be built as well. We show in the sequel that the symmetric integral can cope with this example, and not the asymmetric integral. A clear explanation can be obtained by means of the interaction transform and 2-additive measures.

\subsection{Decision with 2-additive capacities}

We begin by the introduction of $k$-additive measures, proposed by Grabisch in connection with the interaction index [11]. The aim was to reduce the exponential complexity implied by the use of a set function, which causes 
the number of parameters to be $2^{n}$ for a $n$ criteria problem. It has been shown that the interaction transform seems to convey the semantics of the capacity $[9,17]$. Therefore, a natural idea would be to limit the number of non-zero coefficients for $I(A)$, knowing that the most significant coefficients are for a low cardinality of $A$.

Definition $4 \mathrm{~A}$ capacity is said to be a $k$-additive measure (or a $k$-additive capacity) if $I(A)=0$ for all coalitions of more than $k$ criteria, and there exists at least one $A$ of exactly $k$ elements such that $I(A) \neq 0$.

It can be shown that an equivalent definition is obtained if one replaces $I$ by the Möbius transform (it is in fact the usual definition, see [11]). This shows that 1-additive measures are ordinary (additive) measures in the classical sense.

A good compromise seems to be $k=2$, since we can express interaction between pairs of criteria, with still a low complexity. In this case, [10] has shown that the Choquet integral can be expressed as follows, for every $t \in \mathbb{R}_{+}^{n}:$

$$
\mathcal{C}_{v}(t)=\sum_{I_{i j}>0}\left(t_{i} \wedge t_{j}\right) I_{i j}+\sum_{I_{i j}<0}\left(t_{i} \vee t_{j}\right)\left|I_{i j}\right|+\sum_{i=1}^{n} t_{i}\left(\phi_{i}-\frac{1}{2} \sum_{j \neq i}\left|I_{i j}\right|\right),
$$

with the property that $\phi_{i}-\frac{1}{2} \sum_{j \neq i}\left|I_{i j}\right| \geq 0$ for all $i$, where $\phi_{i}=I(\{i\})$ is the Shapley index. It can be seen that the Choquet integral for 2-additive measures can be decomposed in a conjunctive, a disjunctive and an additive part, corresponding respectively to positive interaction indices, negative interaction indices, and the Shapley value. Moreover, due to the normalization $\sum_{i=1}^{n} \phi_{i}=1$, it is easy to see that (20) is a convex combination of disjunctions, conjunctions, and a linear part. Equation (20) permits us to give the following interpretation of the interaction transform:

- a positive interaction index $I_{i j}$ implies a conjunctive behaviour between criteria $i$ and $j$. This means that both scores on $i$ and $j$ have to be high in order to give a significant contribution to the global score. In this sense, these criteria have to be satisfied together, they are complementary.

- a negative interaction index $I_{i j}$ implies a disjunctive behaviour between criteria $i$ and $j$. This means that one of the scores on $i$ and $j$ has to be high in order to give a significant contribution to the global score. In this sense, these criteria do not have to be satisfied together, they are redundant.

- if for some $i, I_{i j}=0$ for all $j$, then criterion $i$ appears only in a linear way into the global score. In this sense, $i$ is independent from the other criteria.

- the Shapley value appears only in the linear part of the global score. It represents the global importance of a criterion in the decision process, exactly as in cooperative game theory the Shapley value expresses the prospect of each player to play the game (or, the power index of each player in a voting problem). 
We turn now to the case of real-valued scores. For the case of the asymmetric integral, due to the fact that there is no change of expression w.r.t $I$ when the integrand takes negative values (see (15)), we see that (20) is still valid. For the case of the symmetric integral, we have the following result.

Proposition 4 Let $t \in \mathbb{R}^{n}$, and $v$ a 2-additive measure. Then

$$
\begin{aligned}
\check{\mathcal{C}}_{v}(t)= & \sum_{i, j \in N^{+}, I_{i j}>0}\left(t_{i} \wedge t_{j}\right) I_{i j}+\sum_{i, j \in N^{-}, I_{i j}>0}\left(t_{i} \vee t_{j}\right) I_{i j} \\
& +\sum_{i, j \in N^{+}, I_{i j}<0}\left(t_{i} \vee t_{j}\right)\left|I_{i j}\right|+\sum_{i, j \in N^{-}, I_{i j}<0}\left(t_{i} \wedge t_{j}\right)\left|I_{i j}\right| \\
& +\sum_{i \in N^{+}} t_{i}\left(\sum_{j \in N^{-}, I_{i j}<0}\left|I_{i j}\right|\right)+\sum_{i \in N^{-}} t_{i}\left(\sum_{j \in N^{+}, I_{i j}<0}\left|I_{i j}\right|\right) \\
& +\sum_{i=1}^{n} t_{i}\left(\phi_{i}-\frac{1}{2} \sum_{j \neq i}\left|I_{i j}\right|\right) .
\end{aligned}
$$

with $N^{+}=\left\{i \in N \mid t_{i} \geq 0\right\}$, and $N^{-}=N \backslash N^{+}$.

Proof: We use Proposition 1 and the fact that $v$ is 2-additive. Also, since $m(\emptyset)=0$ and due to (5), we can replace in (16) the summations $A \subset N^{+}$ and $A \subset N^{-}$by $A \subset N^{+}, A \neq \emptyset$ and $A \subset N^{-}, A \neq \emptyset$. Specifically:

$$
\begin{aligned}
\check{\mathcal{C}}_{v}(t)= & \sum_{i \in N^{+}} t_{i}\left(\phi_{i}-\frac{1}{2} \sum_{\substack{j \neq i \\
I_{i j}>0}} I_{i j}\right)+\sum_{\substack{i, j \in N^{+} \\
I_{i j}>0}}\left(t_{i} \wedge t_{j}\right) I_{i j} \\
& +\sum_{i \in N^{-}} t_{i}\left(\phi_{i}-\frac{1}{2} \sum_{\substack{j \neq i \\
I_{i j}>0}} I_{i j}\right)+\sum_{\substack{i, j \in N^{-} \\
I_{i j}>0}}\left(t_{i} \vee t_{j}\right) I_{i j} \\
& +\sum_{i \in N^{+}} t_{i}\left(\frac{1}{2} \sum_{\substack{j \neq i \\
I_{i j}<0}} I_{i j}\right)+\sum_{\substack{i, j \in N^{+} \\
I_{i j}<0}}\left(t_{i} \vee t_{j}\right)\left|I_{i j}\right|+\sum_{\substack{i \in N^{+} \\
j \in \in N^{-} \\
I_{i j}<0}}\left(t_{i} \vee t_{j}\right)\left|I_{i j}\right| \\
& +\sum_{i \in N^{-}} t_{i}\left(\frac{1}{2} \sum_{\substack{j \neq i \\
I_{i j}<0}} I_{i j}\right)+\sum_{\substack{i, j \in N^{-} \\
I_{i j}<0}}\left(t_{i} \wedge t_{j}\right)\left|I_{i j}\right|+\sum_{\substack{i \in N^{-} \\
j \in \in+\\
I_{i j}<0}}\left(t_{i} \wedge t_{j}\right)\left|I_{i j}\right| .
\end{aligned}
$$

Remarking that

$$
\begin{aligned}
& \sum_{\substack{i \in N^{+} \\
j \in N^{-} \\
I_{i j}<0}}\left(t_{i} \vee t_{j}\right)\left|I_{i j}\right|=\sum_{i \in N^{+}} t_{i} \sum_{j \in N^{-}, I_{i j}<0}\left|I_{i j}\right| \\
& \sum_{\substack{i \in N^{-} \\
j \in N^{+} \\
I_{i j}<0}}\left(t_{i} \wedge t_{j}\right)\left|I_{i j}\right|=\sum_{i \in N^{-}} t_{i} \sum_{\substack{j \in N^{+}, I_{i j}<0\\
}}\left|I_{i j}\right|
\end{aligned}
$$


and regrouping terms, we get the desired result.

This leads to the following interpretation of the interactions in the symmetric integral :

- a positive $I_{i j}$ implies a conjunctive behavior between criteria $i$ and $j$ if the scores for these two criteria are positive, and implies a disjunctive behavior if the scores for the two criteria are negative. This implies that the decision maker changes his behavior when he considers positive or negative scores.

- a negative $I_{i j}$ implies a disjunctive behavior between criteria $i$ and $j$ if the scores for these two criteria are positive, and implies a conjunctive behavior if the scores for the two criteria are negative.

- The Shapley indices appear in the linear part of the sum. We note that the comparison between positive and negative scores are put in the linear part of the symmetric integral. There is no interaction between positive and negative scores.

By contrast, the above results show that the asymmetric Choquet integral follows the same rules whatever the sign of the scores may be. This seems to be a shortcoming for the use of this integral with signed scores, since most of psychologists indeed agree that people act differently when they are face to good things (positive scores) or bad things (negative scores). As a consequence, the symmetric integral seems very attractive thanks to its ability to model the inversion of the decision maker strategy when considering positive or negative scores. The following example illustrates the difference between the two models.

Example 2 We consider the problem of the evaluation of students with respect to three subjects: mathematics, physics and literature. Scores are given from 0 to 20 , and we consider that the scale is bipolar, with neutral element 10. The knowledge about inter-criterion is given under the form of interaction effects:

- scientific subjects are more important, which means that the Shapley value is greater for those criteria.

- redundancy between mathematics and physics, which means that the interaction index is negative for this pair of criteria.

- literature is independent from scientific subjects, which means that the corresponding interaction indices are 0 .

This could be translated as follows (supposing an appropriate methodology):

\begin{tabular}{|l|c|l|c|}
\hline subset $A$ & $I(A)$ & subset $A$ & $I(A)$ \\
\hline Maths & 0.35 & Maths, Physics & -0.5 \\
Physics & 0.35 & Maths, Literature & 0 \\
Literature & 0.3 & Physics, Literature & 0 \\
\hline
\end{tabular}

We consider three students A, B, and C, whose marks and average computed by both symmetric and asymmetric Choquet integrals (using Prop. 4) are given below. 


\begin{tabular}{|c|c|c|c|c|c|}
\hline student & Maths & Physics & Literature & $\mathcal{C}$ & $\mathcal{C}$ \\
\hline A & 16 & 12 & 10 & 13.8 & 13.8 \\
B & 8 & 4 & 10 & 8.2 & 6.2 \\
C & 6 & 6 & 10 & 7.2 & 7.2 \\
\hline
\end{tabular}

We can make the following comments.

- for the asymmetric integral, Maths and Physics are disjunctive in any case, while for the symmetric integral, there is a conjunctive behaviour with $\mathrm{B}$ (negative scores) and disjunctive with $\mathrm{A}$.

- for the asymmetric integral, B is preferred to C (for B: Maths compensates Physics), while for the symmetric integral, $\mathrm{C}$ is better than $\mathrm{B}$ (no compensation occurs).

\section{Acknowledgment}

The authors are indebted to Jean-Claude Vansnick for fruitful and stimulating discussions.

\section{References}

1. A. Chateauneuf and J.Y. Jaffray. Some characterizations of lower probabilities and other monotone capacities through the use of Möbius inversion. Mathematical Social Sciences, 17:263-283, 1989.

2. G. Choquet. Theory of capacities. Annales de l'Institut Fourier, 5:131-295, 1953.

3. A.P. Dempster. Upper and lower probabilities induced by a multivalued mapping. Ann. Math. Statist., 38:325-339, 1967.

4. D. Denneberg. Non-Additive Measure and Integral. Kluwer Academic, 1994.

5. D. Denneberg and M. Grabisch. Interaction transform of set functions over a finite set. Information Sciences, 121:149-170, 1999.

6. D. Dubois, M. Grabisch, F. Modave, and H. Prade. Relating decision under uncertainty and multicriteria decision making models. Int. J. of Intelligent Systems, 15:967-979, 2000.

7. D. Dubois and H. Prade. Possibility Theory. Plenum Press, 1988.

8. D. Dubois and H. Prade. Possibility theory: qualitative and quantitative aspects. In D.M. Gabbay and Ph. Smets, editors, Handbook of Defeasible Reasoning and Uncertainty Management Systems, pages 169-226. Kluwer Academic Publishers, 1998.

9. M. Grabisch. The application of fuzzy integrals in multicriteria decision making. European J. of Operational Research, 89:445-456, 1996.

10. M. Grabisch. Alternative representations of discrete fuzzy measures for decision making. Int. J. of Uncertainty, Fuzziness, and Knowledge Based Systems, 5:587-607, 1997.

11. M. Grabisch. $k$-order additive discrete fuzzy measures and their representation. Fuzzy Sets and Systems, 92:167-189, 1997.

12. M. Grabisch. On the representation of $k$-decomposable measures. In 7th IFSA World Congress, Prague, Czech Republic, June 1997. 
13. M. Grabisch. The interaction and Möbius representations of fuzzy measures on finite spaces, $k$-additive measures: a survey. In M. Grabisch, T. Murofushi, and M. Sugeno, editors, Fuzzy Measures and Integrals - Theory and Applications, pages 70-93. Physica Verlag, 2000.

14. M. Grabisch and Ch. Labreuche. To be symmetric or asymmetric? A dilemna in decision making. In J. Fodor, B. De Baets, and P. Perny, editors, Preferences and Decisions under Incomplete Knowledge, pages 179-194. Physica Verlag, 2000 .

15. M. Grabisch and Ch. Labreuche. The Šipoš integral for the aggregation of interacting bipolar criteria. In 8th Int. Conf. on Information Processing and Management of Uncertainty in Knowledge-Based Systems (IPMU), pages 395-401, Madrid, Spain, July 2000.

16. M. Grabisch, Ch. Labreuche, and J.C. Vansnick. On the extension of pseudoBoolean functions for the aggregation of interacting bipolar criteria. European J. of Operational Research, submitted.

17. M. Grabisch and M. Roubens. Application of the Choquet integral in multicriteria decision making. In M. Grabisch, T. Murofushi, and M. Sugeno, editors, Fuzzy Measures and Integrals - Theory and Applications, pages 348-374. Physica Verlag, 2000.

18. D. Kahneman and A. Tversky. Prospect theory: an analysis of decision under risk. Econometrica, 47:263-291, 1979.

19. R.L. Keeney and H. Raiffa. Decision with Multiple Objectives. Wiley, New York, 1976.

20. D.H. Krantz, R.D. Luce, P. Suppes, and A. Tversky. Foundations of measurement, volume 1: Additive and Polynomial Representations. Academic Press, 1971.

21. J.L. Marichal. Aggregation operators for multicriteria decision aid. $\mathrm{PhD}$ thesis, University of Liège, 1998.

22. T. Murofushi. A technique for reading fuzzy measures (I): the Shapley value with respect to a fuzzy measure. In 2nd Fuzzy Workshop, pages 39-48, Nagaoka, Japan, October 1992. In Japanese.

23. T. Murofushi and S. Soneda. Techniques for reading fuzzy measures (III): interaction index. In 9th Fuzzy System Symposium, pages 693-696, Sapporo, Japan, May 1993. In Japanese.

24. D. Schmeidler. Integral representation without additivity. Proc. of the Amer. Math. Soc., 97(2):255-261, 1986.

25. D. Schmeidler. Subjective probability and expected utility without additivity. Econometrica, 57(3):571-587, 1989.

26. G. Shafer. A Mathematical Theory of Evidence. Princeton Univ. Press, 1976.

27. L.S. Shapley. A value for $n$-person games. In H.W. Kuhn and A.W. Tucker, editors, Contributions to the Theory of Games, Vol. II, number 28 in Annals of Mathematics Studies, pages 307-317. Princeton University Press, 1953.

28. M. Sugeno. Theory of fuzzy integrals and its applications. PhD thesis, Tokyo Institute of Technology, 1974.

29. M. Sugeno and T. Murofushi. Fuzzy measure theory, volume 3 of Course on fuzzy theory. Nikkan Kōgyō, 1993. In Japanese.

30. A. Tversky and D. Kahneman. Advances in prospect theory: cumulative representation of uncertainty. J. of Risk and Uncertainty, 1992.

31. J. Šipoš. Integral with respect to a pre-measure. Math. Slovaca, 29:141-155, 1979. 
32. P. Walley. Coherent lower (and upper) probabilities. Technical Report 22, University of Warvick, Coventry, 1981.

33. P. Walley. Statistical Reasoning with Imprecise Probabilities. Chapman and Hall, London, 1991.

\section{A The co-Möbius transform [13]}

Definition 5 Let $v$ be a capacity. The co-Möbius transform of $v$ is defined by:

$$
\check{m}^{v}(A):=\sum_{B \supset N \backslash A}(-1)^{n-|B|} v(B)=\sum_{B \subset A}(-1)^{|B|} v(N \backslash B), \quad \text { forall } A \subset N .
$$

The co-Möbius transform takes its name from the fact that it is closely related to the Möbius transform of the conjugate capacity:

$$
\check{m}^{\bar{v}}(A)=(-1)^{|A|+1} m^{v}(A), \quad \forall A \subset N, A \neq \emptyset .
$$

It is known in Dempster-Shafer theory under the name of "commonality function" [26]), and in possibility theory [7] under the name of "guaranted possibility measure" (see e.g. [8]).

Its relation with $I$ is given by:

$$
\check{m}(A)=\sum_{B \subset N \backslash A} \beta_{|B|}^{|B|} I(A \cup B)
$$

with $\beta_{k}^{l}$ defined by (7).

The next proposition expresses Choquet and Šipoš integrals in terms of the co-Möbius transform, for any point in $\mathbb{R}^{n}$.

Proposition 5 For any $a \in \mathbb{R}^{n}$,

$$
\begin{aligned}
& \mathcal{C}_{v}(a)=\sum_{A \subset N, A \neq \emptyset}(-1)^{|A|+1} \check{m}^{v}(A) \bigvee_{i \in A} a_{i}=\sum_{A \subset N} m^{\bar{v}}(A) \bigvee_{i \in A} a_{i} \\
& \check{\mathcal{C}}_{v}(a)=\sum_{A \cap N^{+} \neq \emptyset}(-1)^{|A|+1} \check{m}^{v}(A) \bigvee_{i \in A} a_{i}+\sum_{A \cap N^{-} \neq \emptyset}(-1)^{|A|+1} \check{m}^{v}(A) \bigwedge_{i \in A} a_{i} \\
& \check{\mathcal{C}}_{v}(a)=\sum_{A \subset N^{+}}(-1)^{|A|+1} \check{m}^{\bar{v}}(A) \bigwedge_{i \in A} a_{i}+\sum_{A \subset N^{-}}(-1)^{|A|+1} \check{m}^{\bar{v}}(A) \bigvee_{i \in A} a_{i},
\end{aligned}
$$

where $N^{+}=\left\{i \in N \mid a_{i} \geq 0\right\}$, and $N^{-}=N \backslash N^{+}$.

Proof: Let $a \in \mathbb{R}^{n}$. We know from (9) that $\mathcal{C}_{v}(a)=-\mathcal{C}_{\bar{v}}(-a)$. Applying (13), (21), and remarking that $m^{\bar{v}}(\emptyset)=0$, we get:

$$
\begin{aligned}
-\mathcal{C}_{\bar{v}}(-a) & =-\sum_{A \subset N, A \neq \emptyset} m^{\bar{v}}(A) \bigwedge_{i \in A}\left(-a_{i}\right) \\
& =\sum_{A \subset N, A \neq \emptyset}(-1)^{|A|+1} \check{m}^{v}(A) \bigvee_{i \in A} a_{i} .
\end{aligned}
$$


We turn now to the Šipoš integral. By definition, $\check{\mathcal{C}}_{v}(a)=\mathcal{C}_{v}\left(a^{+}\right)-\mathcal{C}_{v}\left(a^{-}\right)$, with $a^{+}, a^{-}$defined as before. Applying (23), we get:

$$
\begin{aligned}
\check{\mathcal{C}}_{v}(a) & =\sum_{A \cap N^{+} \neq \emptyset}(-1)^{|A|+1} \check{m}^{v}(A) \bigvee_{i \in A} a_{i}^{+}-\sum_{A \cap N^{-} \neq \emptyset}(-1)^{|A|+1} \check{m}^{v}(A) \bigvee_{i \in A} a_{i}^{-} \\
& =\sum_{A \cap N^{+} \neq \emptyset}(-1)^{|A|+1} \check{m}^{v}(A) \bigvee_{i \in A} a_{i}+\sum_{A \cap N^{-} \neq \emptyset}(-1)^{|A|+1} \check{m}^{v}(A) \bigwedge_{i \in A} a_{i} .
\end{aligned}
$$

For the second expression, we start from (14), and applying (21) again, we get the desired result.

Let us remark that equation (23) permits us to derive a decomposition of capacities on the basis of the conjugate of unanimity games. Specifically, for any $A \subset N$, let us define the vector $\left(1_{A}, 0_{A^{c}}\right):=\left[\delta_{1}(A), \ldots, \delta_{n}(A)\right]$ in $\mathbb{R}^{n}$ by $\delta_{i}(A)=1$ iff $i \in A$, and 0 otherwise. Then by (23), we have:

$$
\begin{aligned}
\mathcal{C}_{v}\left(1_{A}, 0_{A^{c}}\right) & =\sum_{B \cap A \neq \emptyset}(-1)^{|B|+1} \check{m}^{v}(B) \\
& =\sum_{B \subset N, B \neq \emptyset}(-1)^{|B|+1} \check{m}^{v}(B) \bar{u}_{B}(A),
\end{aligned}
$$

since $\bar{u}_{B}(A)=1$ iff $A \cap B \neq \emptyset$, and 0 otherwise. Since $\mathcal{C}_{v}\left(1_{A}, 0_{A^{c}}\right)=v(A)$ (this is immediate from equation (8)), and using the second equality in (23), we have the following decomposition formula (compare with (2)):

$$
v(A)=\sum_{B \subset N, B \neq \emptyset}(-1)^{|B|+1} \check{m}^{v}(B) \bar{u}_{B}(A)=\sum_{B \subset N} m^{\bar{v}}(B) \bar{u}_{B}(A) .
$$

